\title{
PATRONES DE DISTRIBUCIÓN EN LA FLORA LIQUÉNICA XERÓFILA DEL SURESTE DE ESPAÑA
}

\author{
José M. EGEA y F. Leandro ALONSO
}

\begin{abstract}
RESUMEN. Patrones de distribución en la flora liquénica xerófila del sureste de España. Se hace un análisis fitogeográfico de la flora liquénica de zonas subáridas del sureste de España, la más seca de Europa. Se han distinguido los patrones de distribución siguientes: Xerotérmico-Pangeico, Circumtético, Mesogeo, Mediterráneo, Oeste Mediterráneo-Macaronésico, Iberoafricano, Mediterráneo-Sudafricano. 6 taxones son, según los datos que disponemos en la actualidad, endémicos del área considerada.

Se comenta la posible diferenciación geográfica e histórica de cada elemento y se presentan mapas de distribución con algunas de las especies más representativas de cada grupo.
\end{abstract}

Palabras clave. Biogeografía, elementos xerófilos, flora liquénica, sureste España.

ABSTRACT. Distribution patterns in the xerophilous lichen flora of the southeastern Spain. A phytogeographic analisis of lichen flora of subarides zones of southeastern Spain, the dryest in Europe, has been carried out. The following xerophilous distribution patterns has been recognized: XerothermicPangaean, Circumtethyan, Mesogean, Mediterranean, West Mediterranean, Iberoafrican, MediterraneanSouthafrican. According to the available data, 6 taxa are endemic in this area.

The hipothetic geographical and historical differentiation of each phytogeographycal element is discussed. Maps of some representative species belonging to each distribution pattern are presented.

Key words. Biogeographic, xeric element, lichen flora, southeastern Spain.

\section{INTRODUCCIÓN}

El sureste de España se encuentra enclavado en un área biogeográfica singular, lo que ha permitido el desarrollo de una flora muy rica e interesante. Se estima que en este territorio se desarrolla, aproximadamente, un tercio de las especies vegetales que viven en la
Península Ibérica. Esta riqueza florística se justifica por haber actuado esta zona, de clima invernal muy suave, como refugio de especies muy termófilas y por presentar una gran diversidad geológica, geomorfológica y altitudinal.

Entre la flora cormofítica, predominan especies de distribución mediterránea, muy

Realizado en el marco del proyecto de investigación PB 93-1129-C02-01, financiado por la Dirección General de Investigación Científica y Técnica (DGICYT) 
termófilas y xerófilas, con un elevado número de endemismos e iberoafricanismos. La flora liquénica está integrada por numerosos elementos boreomediterráneos, cosmopolitas o subcosmopolitas. No obstante, existe un elevado número de epecies termófilas y xerófilas, con una distribución más o menos amplia en otras zonas secas de la Tierra.

Por su interés biogeográfico e histórico, creemos interesante estudiar los principales patrones de distribución que podemos reconocer en la flora liquénica xerófila del sureste de España.

\section{MÉTODO}

El área de distribución de cada especie se ha elaborado a partir de los datos extraídos en obras generales (Clauzade \& Roux 1985, Egan 1987, Galloway 1985, Purvis \& al. 1992, etc.), y de algunas revisiones monográficas recientes, como: Anema-Thyrea-Peccania (Moreno y Egea 1992a), Diploschistes (Lumbsch 1989), Dirina (Tehler 1983), Catapyrenium (Breuss 1990), Heppia-Peltula (Egea 1989a), Lecanographa (Egea y Torrente 1994), Lichinella (Moreno y Egea 1992b), Rinodina (Mayrhofer 1984, Mayrhofer et al. 1994). Se ha tenido en cuenta también otros trabajos que se indican en el texto. Para la nomenclatura hemos seguido en general a Clauzade \& Roux (1985) y Nimis (1993).

Las unidades fitogeográficas se han establecido de acuerdo con los patrones observados en hongos liquenizados y en briófitos.

\section{RESULTADOS}

La inclusión de los taxones en los diferentes patrones hay que tomarla como provisional, debido a que se conoce muy poco de la distribución de algunas especies, y a que quedan muchas áreas por estudiar. a) Elemento Xerotérmico-Pangeico (Frey \& Kürschner 1988): Se incluyen en este elemento los taxones que se encuentran en zonas áridas, subáridas y secas de ambos hemisferios. Su área coincide con sabanas y praderas subtropicales, formaciones leñosas esclerófilas, silvoestepas, estepas, semidesiertos y desiertos cálidos o fríos.

La diferenciación histórica y geográfica de este elemento debió producirse entre finales del Paleozoico (Pérmico superior) y primera mitad del Mesozoico (Trías inferior y medio), hace unos 200-240 millones de años. Durante este periodo, las masas continentales formaban una unidad y se había producido una desecación general del clima, lo que favoreció el desarrollo y expansión por toda la tierra de grupos vegetales xeromorfos.

Algunos de los taxones de este elemento son: Acarospora nodulosa var. reagens (fig. 1), Diploschistes diacapsis, D. ocellatus, Heppia lutosa, Peltula euploca, P. obscurans, $P$. omphaliza, $P$. patellata y $P$. placodizans.

b) Elemento Circumtético (Frey \& Kürschner 1988): Este patrón de distribución lo presentan los taxones que se extienden por las Regiones: Madreana, MediterráneoMacaronésica, Saharo-Arábiga, IranoTuraniana. De forma puntual, pueden penetrar en zonas secas de las regiones adyacentes. Coincide con el área Madreano-Tethyana (sensu Raven \& Axelrod 1974, Barreno 1991). En este área se encuentran formaciones leñosas esclerófilas, silvoestepas, estepas, semidesiertos y desiertos cálidos o fríos del Hemisferio Norte.

La diferenciación histórica y geográfica principal de este elemento puede situarse en el Trías-Jurásico, hace unos 180 millones de años, cuando la Tierra estaba separada por el mar Tethys en dos grandes continentes (Laurasia y Gondwana) y anterior a la formación del ${ }^{\text {. }}$ Atlántico norte.

Entre los elementos circumtéticos 


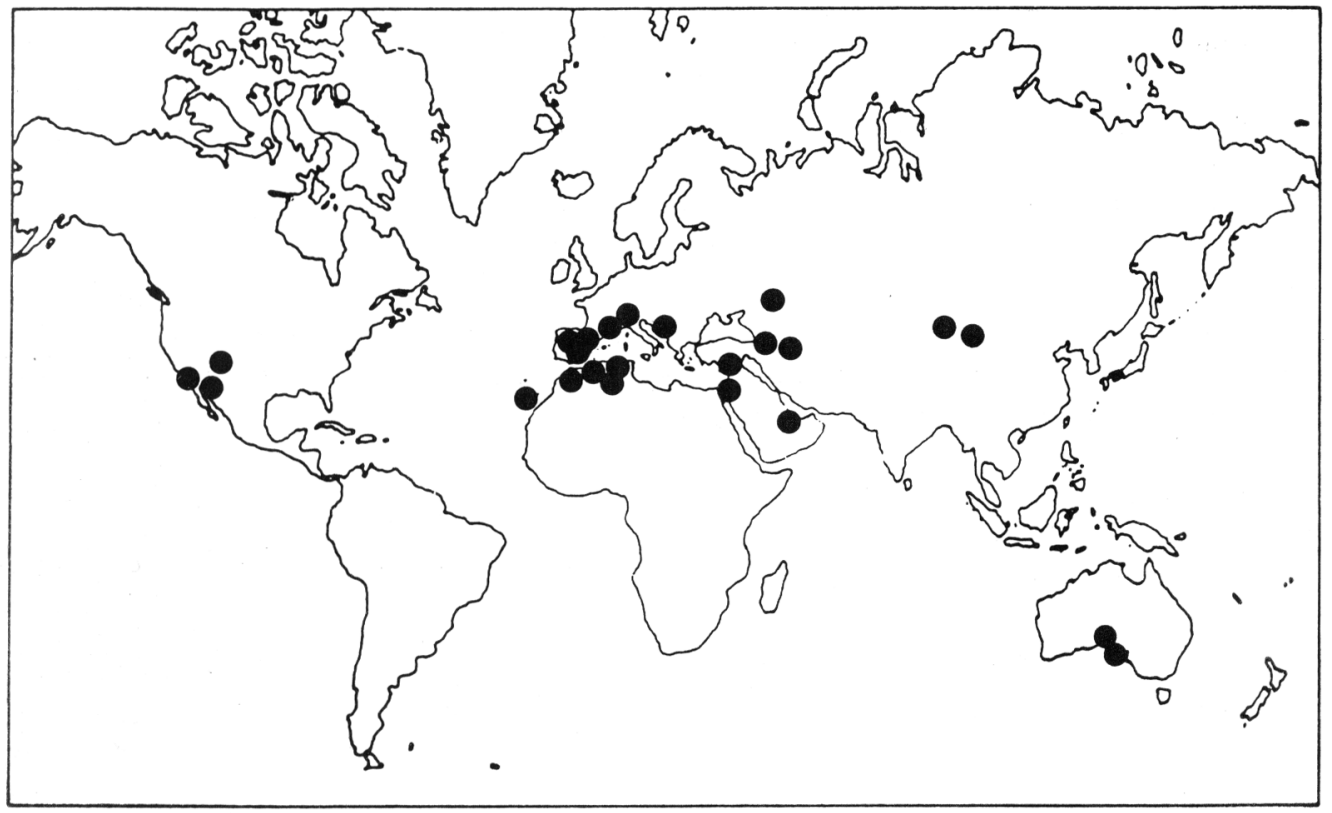

Figura 1. Distribución conocida de Acarospora nodulosa. Known distribution of Acarospora nodulosa.

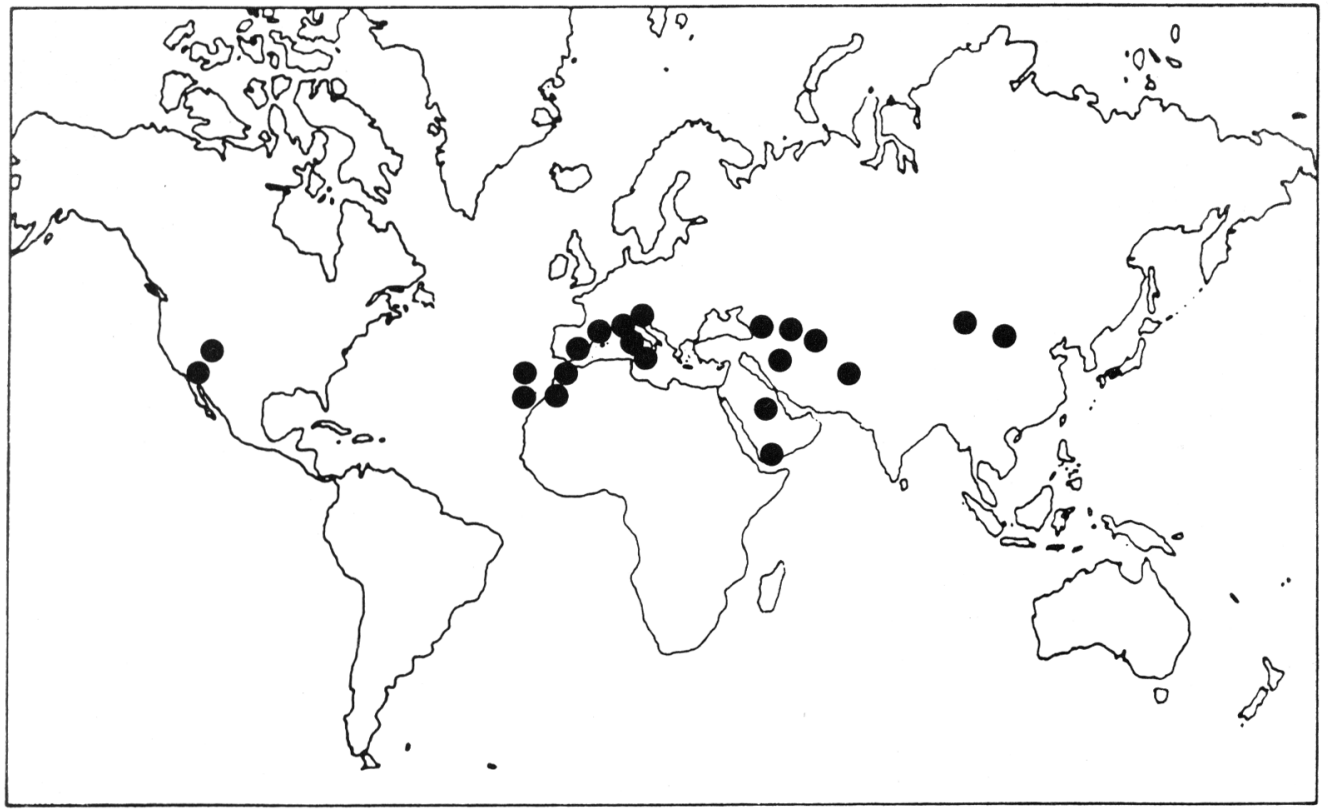

Figura 2. Distribución conocida de Acarospora heufleriana (inc. A. lavicola). Known distribution of Acarospora heufleriana (inc. A. lavicola). 
encontrados en el sureste de España se puede mencionar: Acarospora clauzadeana, A. heufleriana (fig. 2), A. scotica, Buellia fimbriata (fig. 3), Catapyrenium lacinulatum var. latisporum, Dimelaena radiata (Llimona 1975, Sheard 1977, Kärnefelt 1980), Fulgensia desertorum (Barreno 1991), Lichinella cribellifera, L. stipatula (Henssen 1968), Opegrapha ochrocincta, Solenopsora holophaea, Thelopsis isiaca y Thyrea girardii.

c) Elemento Mesogeo (Quézel 1978, 1985, Frey \& Kürschner 1988): La flora Mesogea se restringe a zonas más o menos áridas de Eurasia y norte de Africa. Su área comprende las Regiones siguientes: Mediterránea, Saharo-Arábiga e IranoTuraniana. Coincide con las mismas formaciones vegetales que el elemento anterior.

El desarrollo de este elemento probablemente está relacionado con la xerofitización asociada a la orogénesis del Terciario, hace unos 65 millones de años, y posterior a la apertura del Atlántico norte, que segregó la flora del Tethys entre la flora del sur de Eurasia o Mesogea y la flora del SW de América del Norte o Madreana.

En el sureste de España, este elemento está representado por: Acarospora laqueata (Roux 1991), Buellia zoharyi (fig. 4), Caloplaca alociza, Catapyrenium contumescens, C. semaforonense, Dirina immersa (Egea 1989b), D. paradoxa ssp. africana (Egea 1989b, Follmann 1993), Fulgensia fulgida, Heppia echinulata, $H$. solorinoides, $H$. turgida, Lichinella algerica, L. sinaica, Psora saviczii (Barreno 1991), Placolecis opaca (Hertel 1977), Rinodina guzzinii y Teloschistes lacunosus (fig. 5).

d) Elemento Mediterráneo: Constituye gran parte de la flora del SE de España. Su distribución coincide con el área de los bosques y matorrales esclerofilos, y con los cardonales y tabaibales de la Región Mediterránea (incluida la Región Macaronésica).

Probablemente, este elemento comenzó su desarrollo en el Terciario medio, a partir de la flora artoterciaria, como una adaptación a un período de sequía causado por la pérdida de continuidad del mar Mediterráneo con el océano Atlántico y por el aumento de la aridez que alcanzó el máximo a finales del Mioceno. En esta época, la configuración de los continentes era similar a la actual.

Ejemplos de este elemento son: Acarospora epithallina, A. hilaris (fig. 6), A. microcarpa, Anema nummularium, Arthonia meridionalis (Roux \& Egea 1992), A. oligospora, Aspicilia coronata, Bactrospora patellarioides (Egea y Torrente 1993), Buellia sequax, B. glaucoatra, Caloplaca biatorina ssp. gyalolechioides, C. carphinea, Catapyrenium divisum, C. virescens, Dirina ceratoniae, Lecania spadicea (Mayrhofer 1988, Roux 1991), Lichinella iodopulchra, Lithothelium triseptatum, Opegrapha celtidicola (Torrente \& Egea 1989), O. durieui (Roux \& Egea 1992), O. variaeformis, Petractis luetkemuelleri, P. thelotremella, Porina oleriana, Protoparmelia montagnei, Ramalina requienii (Egea 1989b), Rinodina alba, $R$. beccariana, $R$. santorinensis, Solenopsora cesatii, S. olivacea, Squamarina periculosa (Poelt \& Krüger 1970), Topelia heterospora (fig. 7), T. rosea, Verrucaria cazzae (Roux 1991) y V. limborioides.

e) Elemento Macaronésico-Oeste Mediterráneo (eumediterráneo según Barreno 1991): Incluye los taxones con área restringida a las subregiones Macaronésica y MediterráneoOccidental. Ambas zonas, durante el enfriamiento progresivo de la Tierra en el Terciario superior y los periodos glaciares del Cuaternario, sirvieron de refugio a numerosas especies artoterciarias, las cuales constituyen este interesante subelemento relíctico.

Algunos ejemplos de este subelemento son: Arthothelium crozalsianum, Buellia 


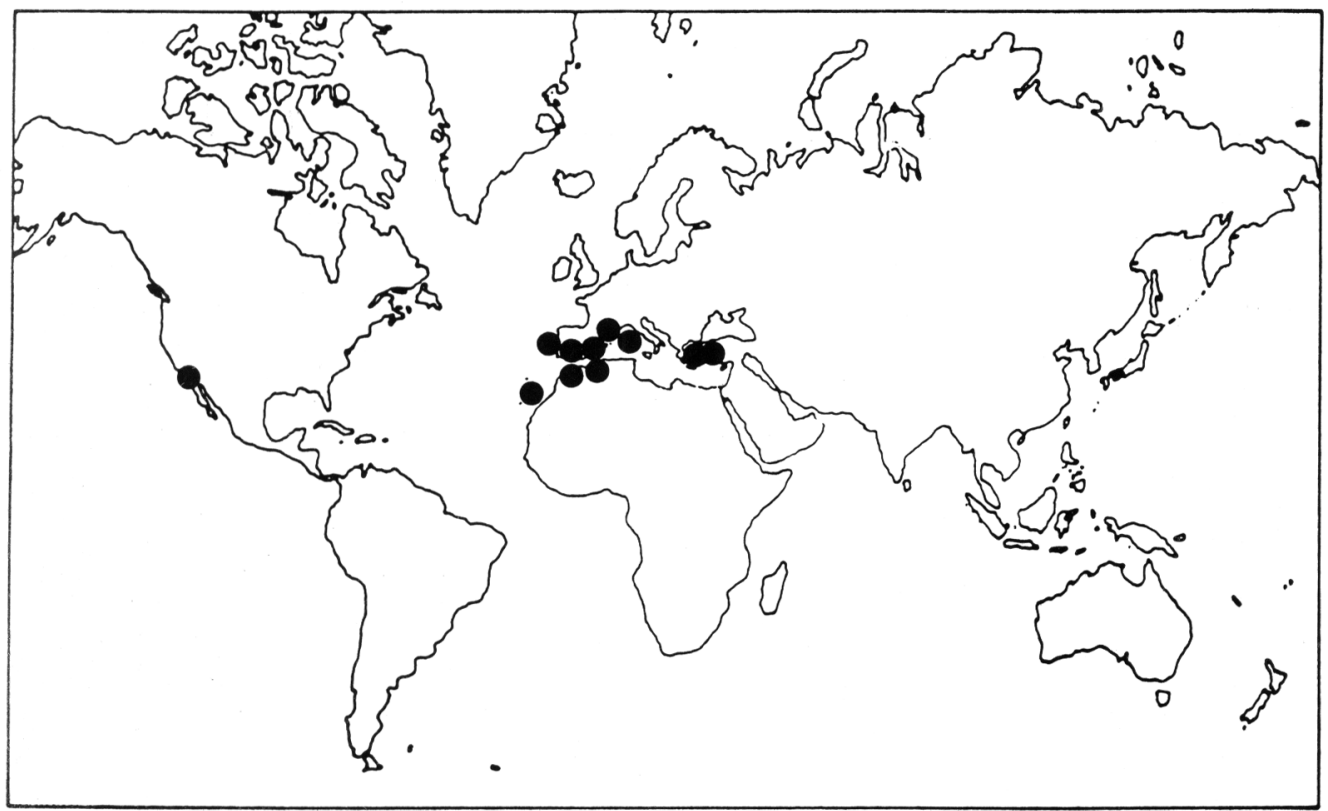

Figura 3. Distribución conocida de Buellia fimbriata. Known distribution of Buellia fimbriata.

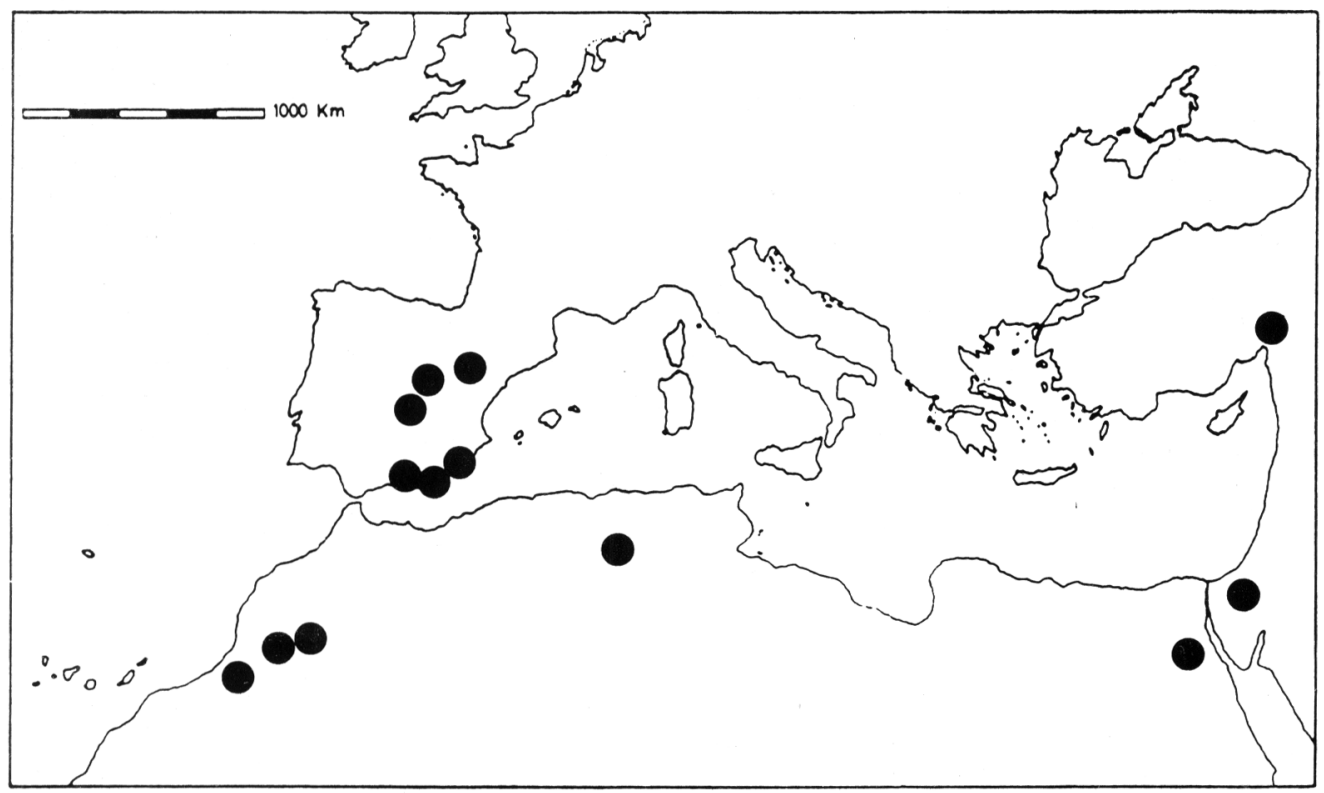

Figura 4. Distribución conocida de Buellia zoharyi. Known distribution of Buellia zoharyi 
sardiniensis, Caloplaca aetnensis (Barreno 1991), C. concinerascens, C. gloriae (Llimona \& Werner 1975), C. ligustica, C. navasiana, $C$. necator, C. subochracea (Roux 1991), C. tavaresiana, Candelariella oleaginescens, Catapyrenium imbricatum, Diploicia subcanescens (Llimona et al. 1976), Gyalecta schisticola (fig. 8), Ingaderia troglodytica, Lecanographa subgrumulosa, Lecanora agardhiana ssp. catalaunica, L. sulphurella, Lichinella robusta, Llimonaea occulta, Peccania cerebriformis, Pertusaria gallica (fig. 9), Pyrenopsis triptococca (Moreno \& Egea 1994), Ramalina clementeana, $R$. rosacea (Egea 1989b), R. tingitana, Rhizocarpon lusitanicum, Roccella canariensis (Egea 1989b, Follmann 1993), R. vicentina (Egea 1989b), Toninia toepferi (Timdal 1991) y Xanthoria resendei (Llimona 1975).

f) Elemento Iberoafricano: Puede considerarse como otro sublelemento mediterráneo, integrado por aquellos taxones que se encuentran en zonas áridas, subáridas y secas del Magrheb y zonas subáridas de la Península Ibérica. De forma puntual, llega a zonas secas del SW de Francia e Italia.

De acuerdo con Blanca (1993), el intercambio de la flora entre el sur de Europa y el norte de Africa fue posible durante el Mioceno superior y el Plioceno, cuando ambos continentes estaban conectados por puentes continentales.

Ejemplos de taxones iberoafricanos son: Acarospora charidema, A. maroccana (fig. 10), Anema nodulosum, A. prodigulum, Heppia gigantea, Peccania fontqueriana, Peltula crispatula, P. obscuratula y Thyrea plicatissima.

\section{g) Elemento Mediterráneo-}

Sudafricano: Hay un grupo de especies integrado por Caloplaca scoriophila (Fig. 11), Glyphopeltis ligustica (Egea et. al., en prensa), Lecanographa grumulosa, L. lyncea,
Opegrapha lutulenta y Sclerophyton circumscripum (fig. 12), que se encuentran en puntos de la región Mediterránea y Sudáfrica. Lecanographa grumulosa, L. lyncea y Sclerophytom circumscriptum se encuentran también en la región Madreana. Un patrón similar (sin incluir la región Madreana) se encuentra en otros grupos de plantas como briófitos (Frey \& Kürschner 1993) y plantas vasculares (Blanca 1993).

La conexión entre ambas áreas pudo producirse a través de las costas del Mar Rojo, Península Arábiga, Somalia, Tanzania y Zambia. Esta zona ha sido bautizada por algunos autores (Blanca 1993) como el «corredor árido». Quézel (1985) indicó que la presencia de géneros pares (plantas vasculares) entre ambas áreas sugiere un disyunción antigua, probablemente del Mioceno.

h) Endemismos: Entre los líquenes, a diferencia de lo que ocurre con las plantas vasculares, son raras las especies endémicas de áreas reducidas. En muchos casos, se trata de especies descritas recientemente, por lo que es probable que presenten áreas de distribución más amplias pero desconocidas en la actualidad.

Taxones con área de distribución restringida a algunos puntos de la Península Ibérica, y que se encuentran en el sureste de España son: Acarospora placodiformis (Llimona 1974), Buellia almeriensis, Fulgensia poeltii (Barreno 1991), Anema suffruticosum y Caloplaca furax.

\section{ANEXO 1}

Se indican en orden alfabético todas las especies citadas en el texto, indicando entre paréntesis el patrón de distribución que cada una de ellas presentan.

Acarospora charidema (Clem. ex Colmeiro) Llimona (f)

Acarospora clauzadeana (Llimona) Casares \& Hafellner (b) 


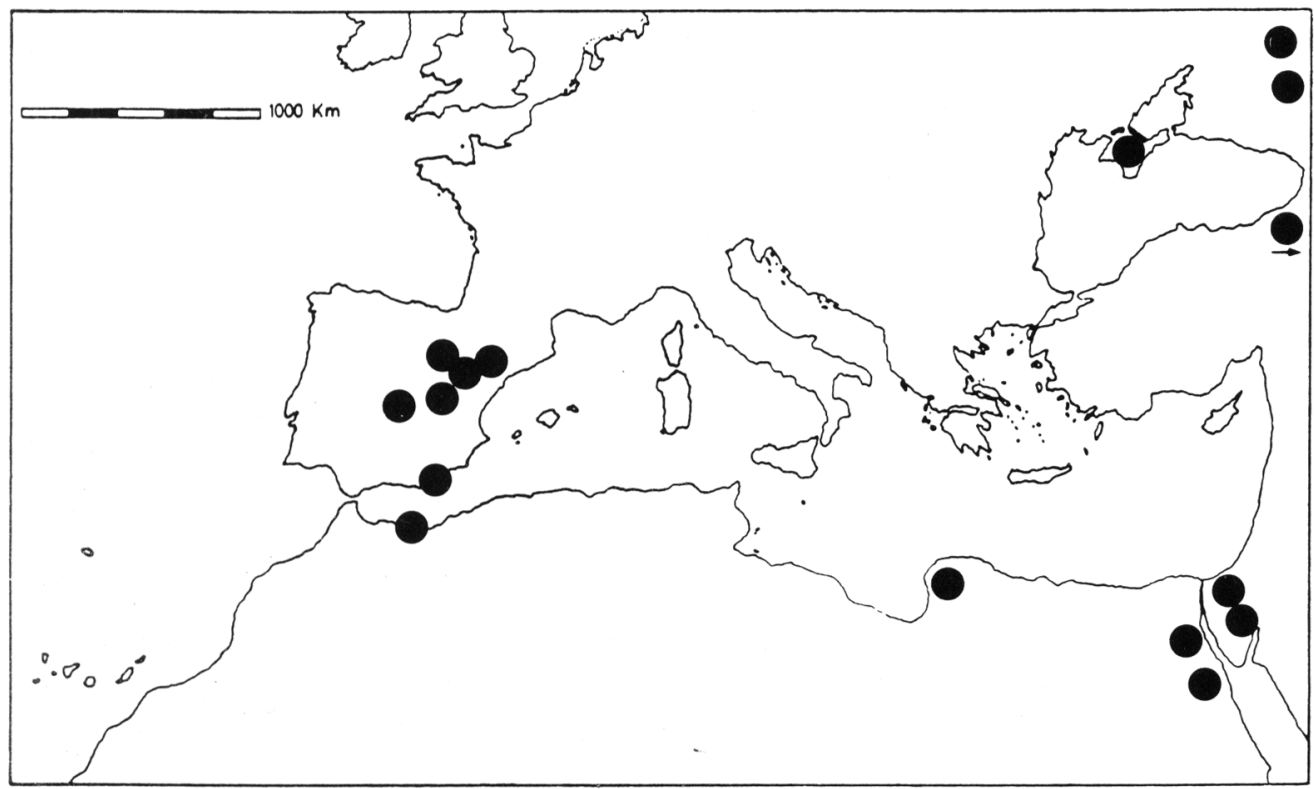

Figura 5. Distribución conocida de Teloschistes lacunosus. Known distribution of Teloschistes lacunosus

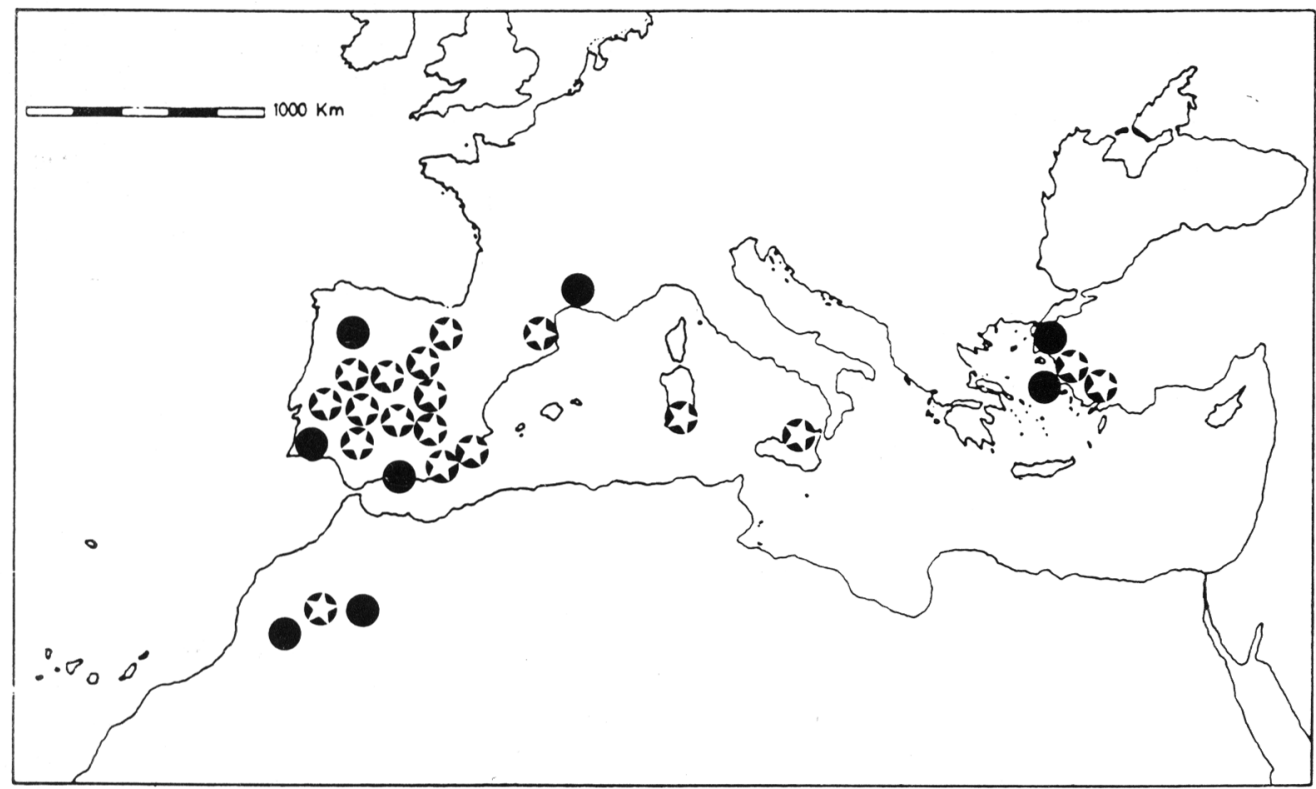

Figura 6. Distribución conocida de Acarospora hilaris (círculo negro); A. epithallina y A. hilaris (estrella). Known distribution of Acarospora hilaris, A. epithallina and A. hilaris. 
Acarospora epithallina Magnusson (d) Acarospora heufleriana Körber (b) Acarospora hilaris (Dufour) Hue (d) Acarospora laqueata Flagey (c) Acarospora maroccana B. de Lesd. (f) Acarospora microcarpa (Nyl.) Wedd. (d) Acarospora nodulosa (Zahlbr.) Clauz. \& Roux var. reagens (Zahlbr.) Clauz. \& Roux (a) Acarospora placodiiformis Magnusson (h) Acarospora scotica Hue (b) Anema nodulosum (Nyl.) Forss. (f) Anema nummularium (Durieu \& Mont.) Nyl. (d) Anema prodigulum (Nyl.) Henssen (f) Anema suffruticosum Moreno \& Egea (h) Arthonia meridionalis Zahlbr. (d) Arthonia oligospora Vezda (d) Arthothelium crozalsianum (B. de Lesd.) B. de Lesd. (e) Aspicilia coronata (Massal.) B. de Lesd. (d) Bactrospora patellarioides (Nyl.) Almq. (d) Buellia almeriensis Llimona (h)

Buellia fimbriata (Tuck.) Sheard (b)

Buellia glaucoatra (Nyl.) Clauz. (d)

Buellia sardiniensis Steiner (e)

Buellia sequax (Nyl.) Zahlbr. (d)

Buellia zoharyi Galum (c)

Caloplaca aetnensis B. de Lesd. (e)

Caloplaca alociza (Massal.) Migula (c)

Caloplaca biatorina (Massal.) Steiner ssp. gyalolechioides (Müll. Arg.) Clauz. \& Roux (d)

Caloplaca carphinea (Fr.) Jatta (d)

Caloplaca concinerascens Nyl. (e)

Caloplaca furax Egea \& Llimona (h)

Caloplaca gloriae Llimona \& Werner (e)

Caloplaca ligustica B. de Lesd. (e)

Caloplaca navasiana Navarro-Rosinés \& Roux (e)

Caloplaca necator Poelt \& Clauzade (e)

Caloplaca scoriophila Zahlbr. (g)

Caloplaca subochracea (Wedd.) Werner (e)

Caloplaca tavaresiana Navarro-Rosinés \& Roux (e)

Candelariella oleaginescens Rondon (e)

Catapyrenium contumescens (Nyl.) Breuss (c)

Catapyrenium divisum (Zahlbr.) Breuss (d)

Catapyrenium imbricatum (Nyl.) Clauz. \& Roux (e)

Catapyrenium lacinulatum (Ach.) Breuss var. latisporum Breuss (b)

Catapyrenium semaforonense Breuss (c)

Catapyrenium virescens (Zahlbr.) Breuss (d)

Dimelaena radiata (Tuck.) Hale.\& Culb. (b)

Diploicia subcanescens (Werner) Hafellner \& Poelt (e)
Diploschistes diacapsis (Ach.) Lumbsch (a)

Diploschistes ocellatus (Vill.) Norman (a)

Dirina ceratoniae (Ach.) Fr. (d)

Dirina immersa Müll. Arg. (c)

Dirina paradoxa (Fée) Tehler ssp. africana (Fée) Tehler (c)

Fulgensia desertorum (Tomin) Poelt (b)

Fulgensia fulgida (Nyl.) Szat. (c)

Fulgensia poeltii Llimona (h)

Glyphopeltis ligustica (B. de Lesd.) Timdal (g)

Gyalecta schisticola Werner (e)

Heppia echinulata Marton \& Galum (c)

Heppia gigantea Egea \& Llimona (f)

Heppia lutosa (Ach.) Nyl. (a)

Heppia solorinoides (Nyl.) Nyl. (c)

Heppia turgida (Ach.) Nyl. (c)

Ingaderia troglodytica Feige \& Lumbsch (e)

Lecania spadicea (Flotow) Zahlbr. (d)

Lecanographa grumulosa (Dufour) Egea \& Torrente (g)

Lecanographa lyncea $(\mathrm{Sm}$.) Egea \& Torrente (g)

Lecanographa subgrumulosa.(Egea, Torrente \& Manrique) Egea \& Torrente (e)

Lecanora agardhiana Ach. ssp. catalaunica Clauz. $\&$ Roux (e)

Lecanora sulphurella Hepp (e)

Lichinella algerica (Steiner) Moreno \& Egea (c)

Lichinella cribellifera (Nyl.) Moreno \& Egea (b)

Lichinella iodopulchra (Crozals) Moreno \& Egea (d)

Lichinella robusta Henssen (e)

Lichinella sinaica (Galun \& Marton) Moreno \& Egea (c)

Lichinella stipatula Nyl. (b)

Lithothelium triseptatum (Nyl.) Aptroot (d)

Llimonaea occulta Egea \& Torrente (e)

Opegrapha celtidicola (Jatta) Jatta (d)

Opegrapha durieui Mont. (d)

Opegrapha lutulenta Nyl. (g)

Opegrapha ochrocincta Werner (b)

Opegrapha variaeformis Anzi (d)

Peccania cerebriformis Henssen \& Büdel (e)

Peccania fontqueriana Moreno \& Egea (f)

Peltula crispatula (Nyl.) Egea (f)

Peltula euploca (Ach.) Pisùt ex Poelt (a)

Peltula obscurans (Nyl.) Gyelnik (a)

Peltula obscuratula (Nyl.) Poelt ex Egea (f)

Peltula omphaliza (Nyl. in Eckf.) Wetmore (a)

Peltula patellata (Bagl.) Swinscow \& Krog (a)

Peltula placodizans (Zahlbr.) Wetmore (a)

Pertusaria gallica B. de Lesd. (e)

Petractis luetkemuelleri (Zahlbr.) Vezda (d) 


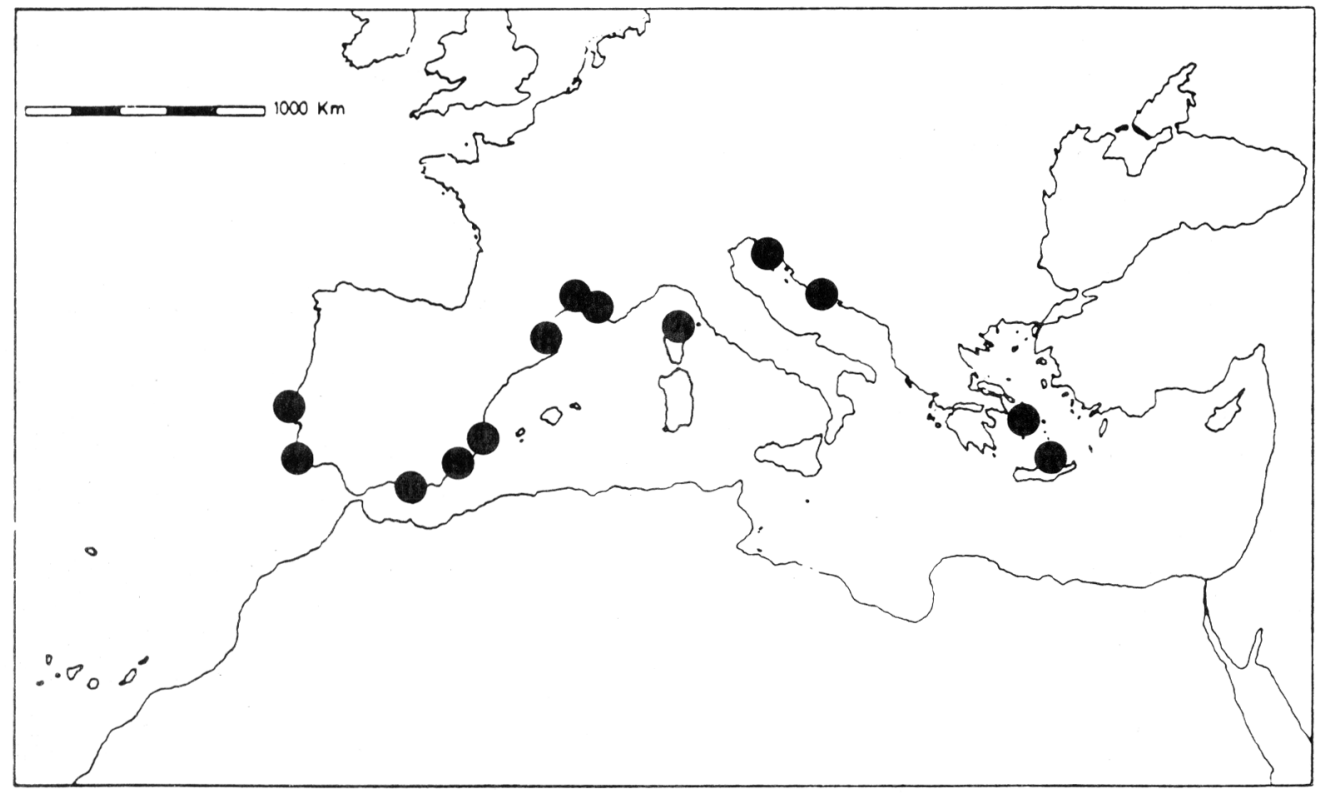

Figura 7. Distribución conocida de Topelia heterospora. Known distribution of Topelia heterospora.

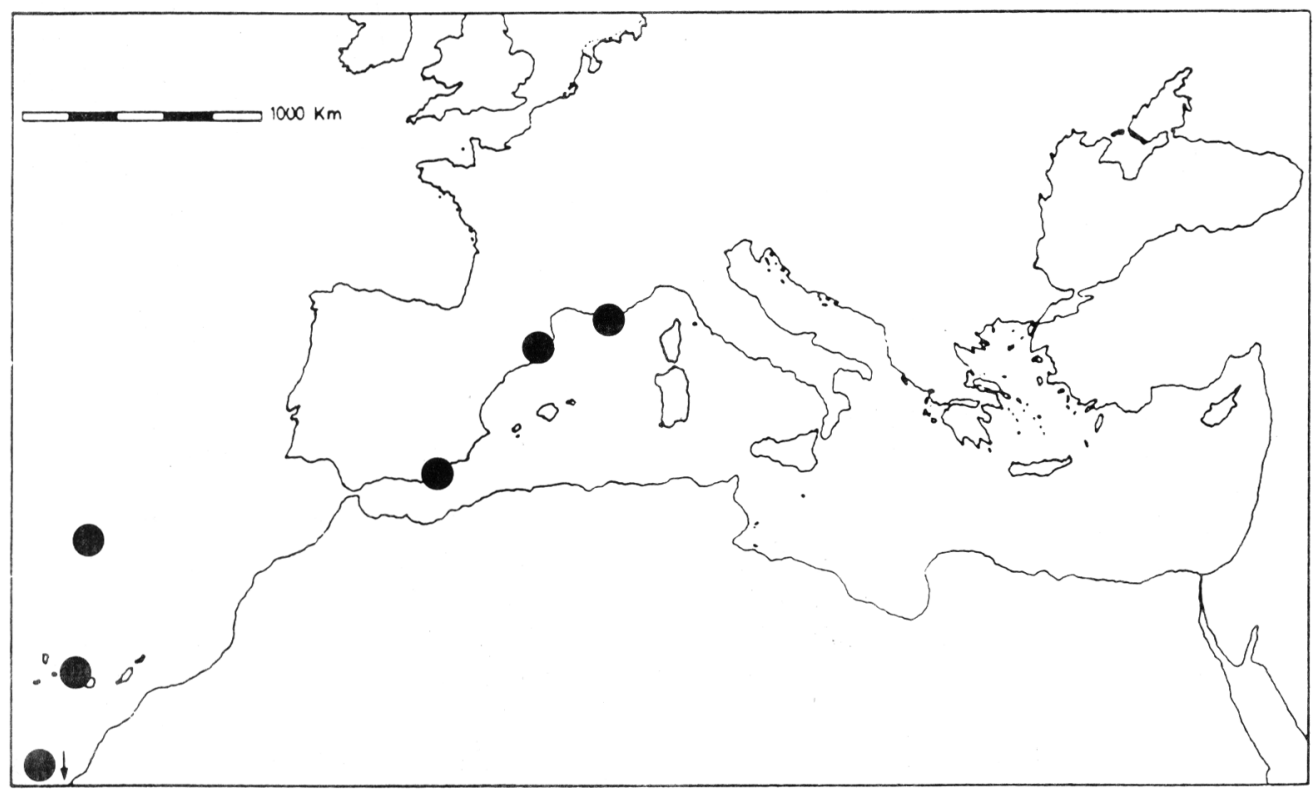

Figura 8. Distribución conocida de Gyalecta schisticola. Known distribution of Gyalecta schisticola. 
Petractis thelotremella (Bagl.) Vezda (d) Placolecis opaca (Dufour ex Fr.) Hafellner (c) Porina oleriana (Massal.) Lettau (d) Protoparmelia montagnei (Fr.) Poelt \& Nimis (d) Psora saviczii (Tomin) Follm. \& Crespo (c) Pyrenopsis triptococca Nyl. (e)

Ramalina clementeana Llimona \& Werner (e) Ramalina requienii (De Not.) Jatta (d) Ramalina rosacea (Schaer. ex Massal.) Hepp (e) Ramalina tingitana Salzm. ex Nyl. (e) Rhizocarpon lusitanicum (Nyl.) Arnold (e) Rinodina alba Metzler ex Arnold (d) Rinodina beccariana Bagl. (d) Rinodina guzzinii Jatta (c) Rinodina santorinensis Steiner (d) Roccella canariensis Darb. em Vainio (e) Roccella vicentina (Vainio) Follmann (e) Sclerophyton circumscripum (Taylor) Zahlbr. (g) Solenopsora cesatii (Massal.) Zahlbr. (d) Solenopsora holophaea (Mont.) G. Samp. (b) Solenopsora olivacea (Fr.) Kilias (d) Squamarina periculosa (Schaerer) Poelt (d) Teloschistes lacunosus (Rupr.) Sav. (c)

Thelopsis isiaca Stizenb. (b) Thyrea girardii (Durieu \& Mont.) Bagl. \& Car. (b) Thyrea plicatissima (Nyl.) Zahlbr. (f)

Toninia toepferi (B. Stein) Navàs (e) Topelia heterospora (Zahlbr.) P. M. Jörg. \& Vezda (d) Topelia rosea (Servít) P. M. Jörg. \& Vezda (d) Verrucaria cazzae Zahlbr. (d) Verrucaria limborioides (Massal.) Clauzade \& Roux (d) Xanthoria resendei Poelt \& Tavares (e)

\section{BIBLIOGRAFÍA}

BARRENO, E. -1991- Phytogeography of terricolous lichens in the Iberian Peninsula and Canary Islands. Bot. Chron., 10: 199-210.

BLANCA, G. -1993-Origen de la Flora Andaluza. En Introducción a la Flora Andaluza. Valdés, B. (ed.). Junta de Andalucía. Consejería de Cultura y Medio Ambiente, Agencia de Medio Ambiente.

BREUSS, O. -1990- Die Flechtengattung Catapyrenium (Verrucariaceae) in Europa. Stapfia, 23: 1-172.

CLAUZADE, G. \& C. ROUX -1985- Likenoj de Okcidenta Europo Ilustrita determinlibro. Bull. Soc. Bot. Centre-Ouest, n.s., 7: 1-893.

EGAN, R. S. -1987- A Fifth Checklist of the Lichen-
Forming Lichenicolous and allied Fungi on the Continental United States and Canada. The Bryologist, 90: 77-173.

EGEA, J. M. -1989a- Los géneros Heppia y Peltula (Líquenes) en Europa Occidental y Norte de Africa. Biblioth. Lichenol., 31: 1-122 (1989). J. Cramer.

EGEA, J. M. -1989b- Las comunidades liquénicas saxícolas, ombrófobas, litorales del suroeste de Europa y norte de Africa (Roccelletea phycopsis classis prov.). Studia Geobotanica, 9: 73-152.

EGEA J.M. y P. TORRENTE -1993- The lichen genus Bactrospora Massal. Lichenologist, 25: 211-255.

EGEA J.M. y P. TORRENTE -1994- El género de hongos liquenizados Lecanactis (Ascomycotina). Biblioth. Lichenol., 54: 1-205.

EGEA J.M., X. LLIMONA y E. MANRIQUE -en prensa- Nuevos datos sobre Glyphopeltis ligustica (Lecanorales) y su área de distribución. Festschrift Follmann,.

FOLLMANN, G. -1993- Vorarbeiten zu einer Monographie der Flechtenfamilie Roccellaceae (Opegraphales, Ascolichenes). IX. Bestandsaufnahme und Verbreitung, Haushalt und Vergesellschaftung, Gefährdung und Schutz der makaronesischen Sippen. Courier Forsch.Inst. Senckenberg, 159: 175-193.

FREY, W. \& H. KÜRSCHNER. -1988- Bryophytes of the Arabian Peninsula and Socotra. Floristics, phytogeography and definition of the Xerothermic Pangaean element. Studies in Arabian bryophytes 12. Nova Hedwigia, 46(12): 37-120.

FREY, W. \& H. KÜRSCHNER -1993Trichostomopsis trivialis (C. Müll.) Robins. (Pottiacea, Musci) eine südafrikanischmediterran disjuntke Sippe, neu für die Bryoflora Jordaniens. Crypt. Bot., 3: 152-156.

GALLOWAY, D. J. -1985- Flora of New Zealand Lichens. P. D. Hasselberg, N. Z. Government Printer, Wellington. $662 \mathrm{pp}$.

HENSSEN A. -1968- Eine neue Lichinella-Art aus Nordamerica (Lichenes). Nova Hedwigia, 15: 543-550.

HERTEL, H. -1977-Gesteinischebewohnende Arten der Sammelgattung Lecidea (Lichenes) aus Zentral-, Ost-, und Südasien. Khumbu Himal., 6(3): 145-378.

KARNEFELT, I. -1980- Lichens of western North 


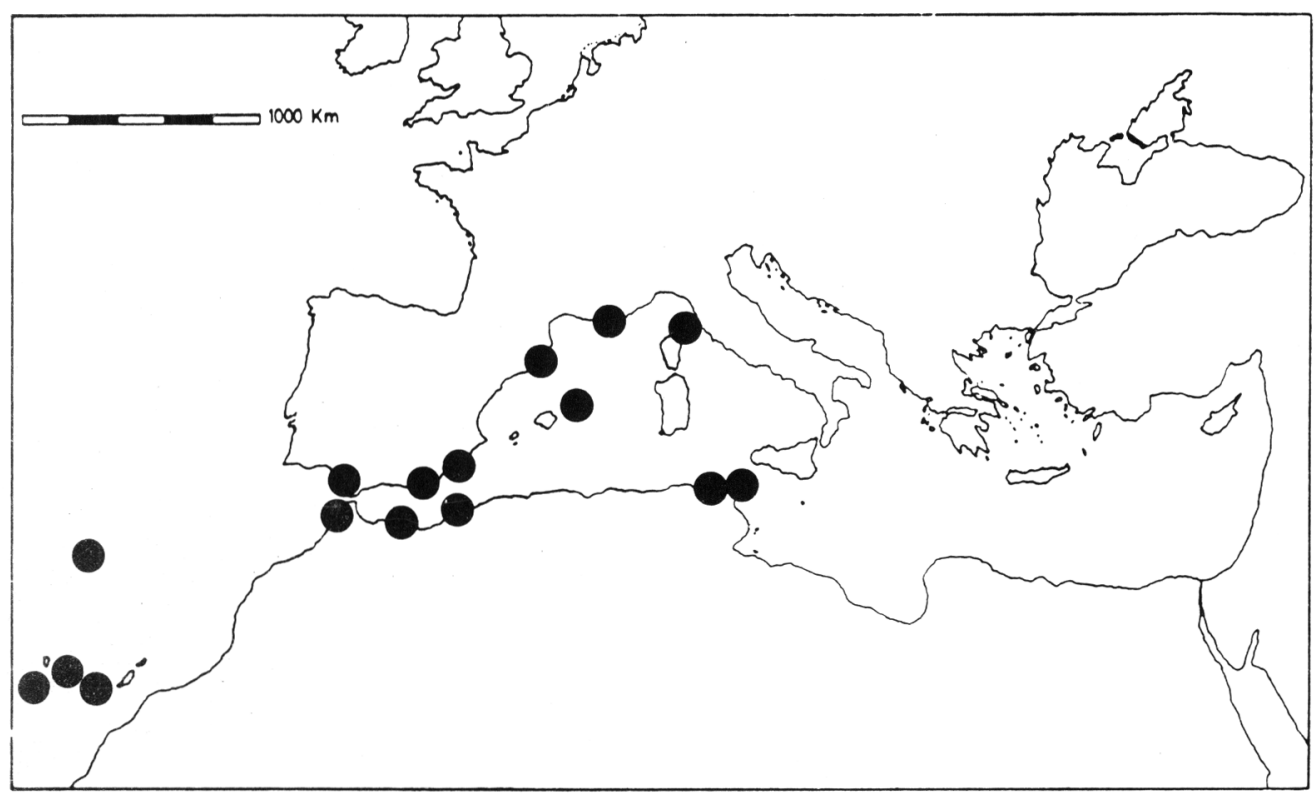

Figura 9. Distribución conocida de Pertusaria gallica. Known distribution of Pertusaria gallica.

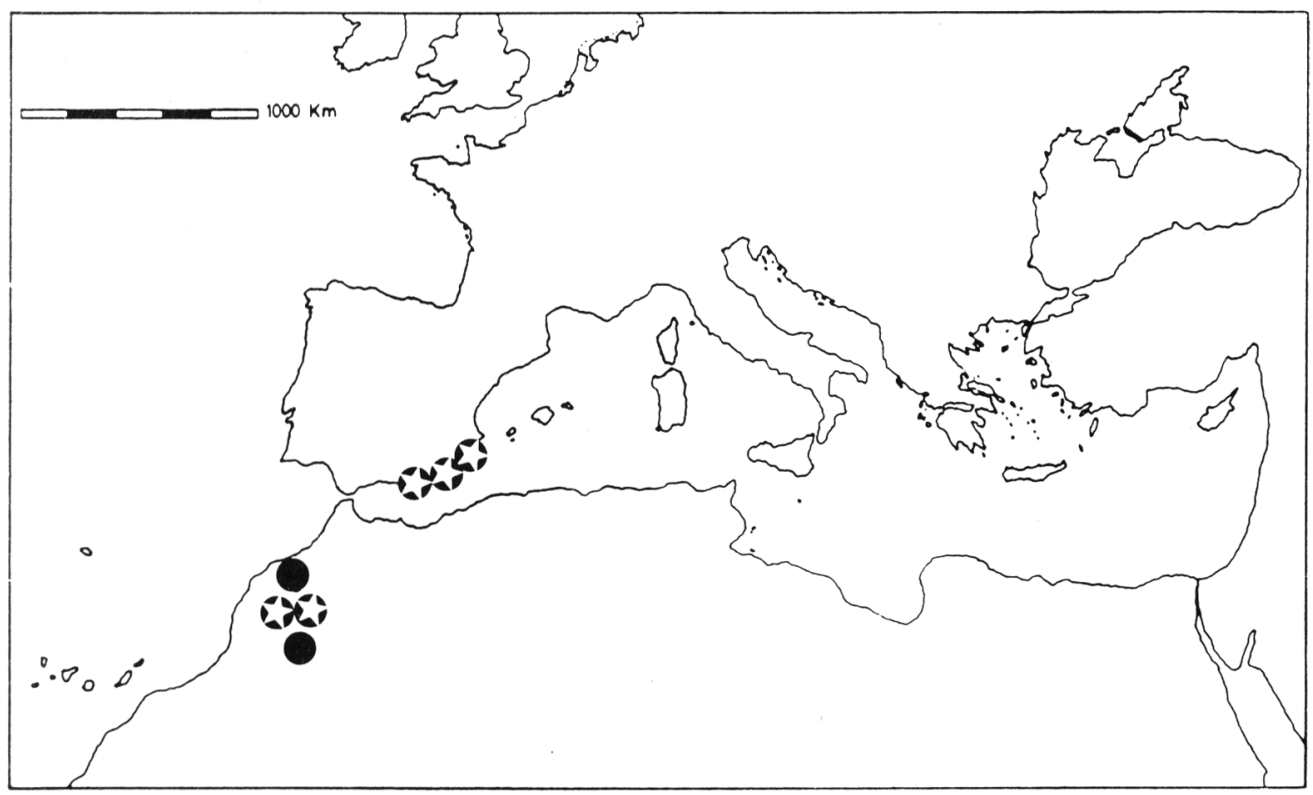

Figura 10. Distribución conocida de Acarospora charidema (círculo negro), A. maroccana y A. charidema (estrella). Known distribution of Acarospora charidema, A. maroccana and A. charidema. 


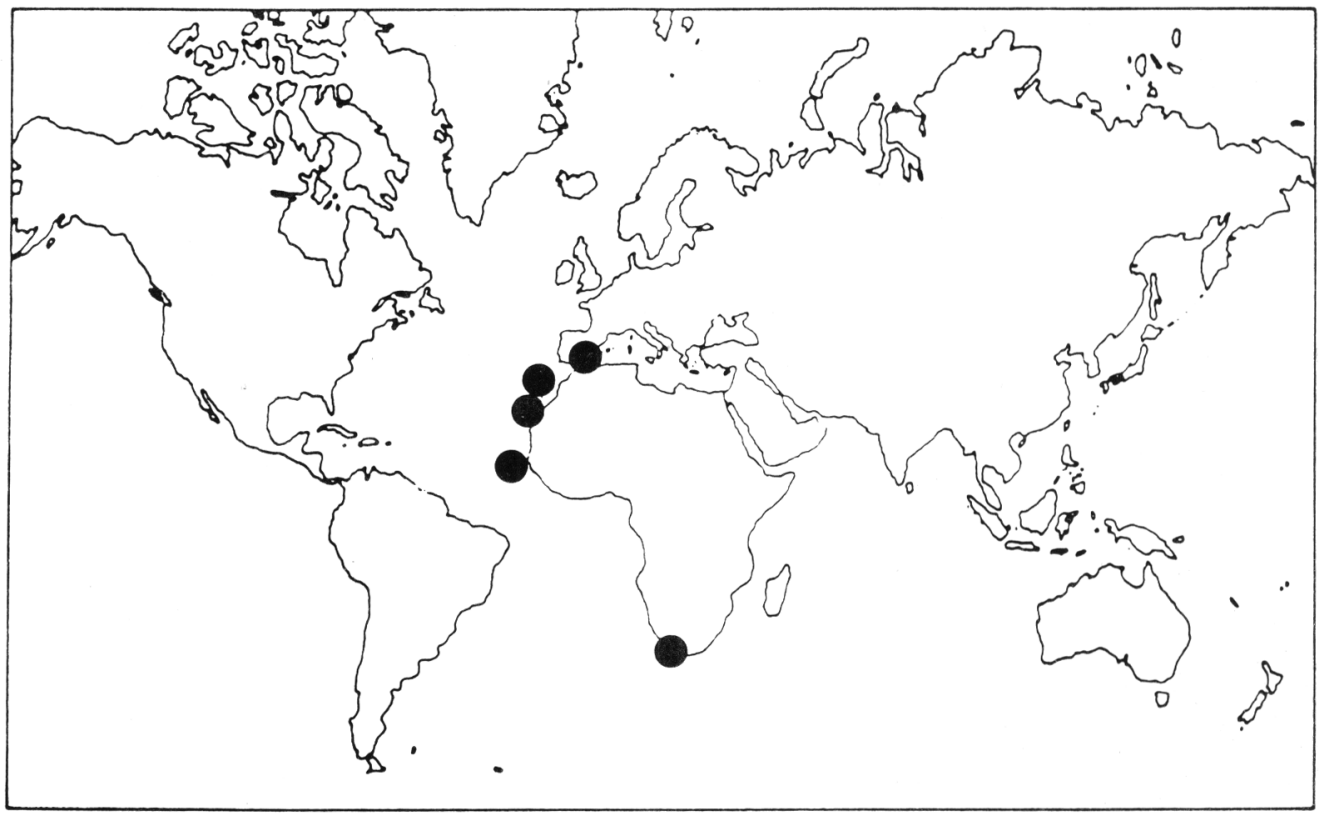

Figura 11. Distribución conocida de Caloplaca scoriophila. Known distribution of Caloplaca scoriophila

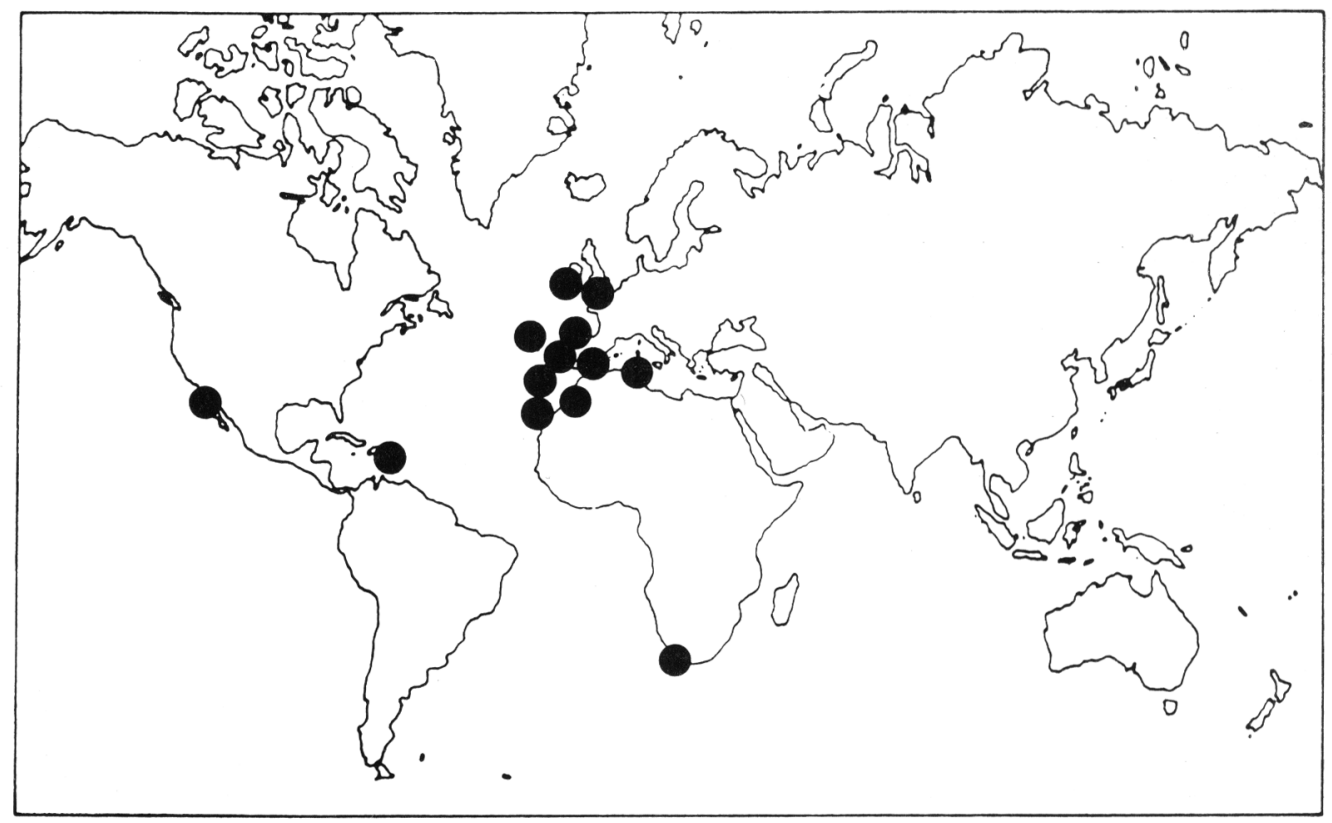

Figura 12. Distribución conocida de Sclerophyton circumscriptum. Known distribution of Sclerophyton circumscriptum. 
America with disjunctions in Macaronesia and West Mediterranean region. Bot. Notiser, 133: 569-577.

LLIMONA, X. -1974- Las comunidades de líquenes de los yesos de España. Resumen Tesis Doctoral, Univ. de Barcelona. 18 pp.

LLIMONA, X. -1975- Xanthoria resendei Poelt et Tavares, en el SE de España; fitosociología y ecología. Anales Inst. Bot. Cavanilles, 32 (2): 909-922.

LLIMONA, X. \& R. G. WERNER -1975- Quelques lichens nouveaux ou intéressants de la Sierra de Gata (Almería, SE de l'Espagne). Acta Phytotax. Barcinonensia, 16: 1-24.

LLIMONA, X., R. G. WERNER, R. LALLEMANT \& J. C. BOISSIERE-1976-A propos du Buellia subcanescens R. G. Werner, espèce primaire du Buellia canescens (Dicks) D. N. Rev. Bryol. Lichénol., 42(1): 617-635.

LUMBSCH, H. T. -1989- Die holarktischen Vertreter der Flechtengattung Diploschistes (Thelotremataceae). Journ. Hattori Bot. Lab., 66: 133-196.

MAYRHOFER, H. -1984- Die Saxicolen Arten der Flechtengattungen Rinodina und Rinodinella in der Alten Welt. Journ. Hattori Bot. Lab., 55: 327-493.

MAYRHOFER, H., M. MATZER, J. SATTLER \& J. M. EGEA -1994- A revision of the AtlanticMediterranean Rinodina beccariana and related taxa (lichenized Ascomycetes, Physciaceae). Nova Hedwigia., 57: 281-304.

MAYRHOFER, M. -1988- Studien über die saxicolen Arten der Flechtengattung Lecania in Europa, II. Lecania s. str. Biblioth. Lichenol., 28: 1-133.

MORENO, P. P. y J. M. EGEA -1992a- Estudios sobre el complejo Anema-Thyrea-Peccania en el sureste de España y norte de Africa. Acta Bot. Barcinonensia, 41: 1-66.

MORENO, P. P. y J. M. EGEA -1992b- El género Lichinella Nyl. en el sureste de España y norte de Africa. Cryptogamie, Bryol. Lichenol., 13(3): 237-259.

MORENO, P. P. y J. M. EGEA -1994- El género Psorotichia y especies próximas en el sureste de España y norte de Africa. Bull. Soc. linn. Provence, 45: 291-308

NIMIS, P. L. -1993- The lichens of Italy. Museo Regionale de Science Naturali. Torino. 897 pp
POELT, J. \& U. KRÜGER -1970- Die Verbreitungsverhältnisse der Flechtengattung Squamarina in Europa. Feddes Rep., 81: 187-201.

PURVIS, O. W., B. J. COPPINS, D. L. HAW KSWORTH, P. W. JAMES \& D. M. MOORE (eds.) - 1992- The Lichen Flora of Great Britain and Ireland. Nat. Hist. Mus. Publ., London. $710 \mathrm{pp}$.

QUÉZEL, P. -1978- Analysis of the flora of Mediterranean and Sahara Africa. Ann. Missouri Bot. Gard., 65: 479-534.

QUÉZEL, P. -1985-Definition of the Mediterranean region and the origin of its flora. En: GómezCampo, C. (ed.): Plant conservation in the Mediterranean area. Dr W. Junk Publishers. Dordrecht.

RAVEN, P. H. \& D. I. AXELROD -1974- Angiosperm biogeography and past continental movements. Ann. Missouri Bot. Gard., 6: 539-673.

ROUX, C. -1991- Phytogéographie des lichens saxicoles-calcicoles d'Europe méditerranéenne. Bot. Chron., 10: 163-178.

ROUX, C. \& J. M. EGEA -1992- L'Opegraphetum durieui Egea et Roux ass. nov., une association lichénique saxicole-calcicole, halophile. Cryptogamie, Bryol. Lichenol., 13: 105-116.

SHEARD, J. W. -1977- Paleogeography, chemistry and taxonomy of the lichenized Ascomycetes Dimelaena and Thamnolia. The Bryologist, 80: 100-118.

TEHLER, A. -1983- The genera Dirina and Roccellina (Roccellaceae). Opera Bot., 70: 1-86.

TIMDAL, E. -1991-A monograph of the genus Toninia (Lecideaceae, Ascomycetes). Opera Bot., 110: 1-137.

TORRENTE, P. \& J.M. EGEA -1989- La familia Opegraphaceae en el área Mediterránea de la Península Ibérica y Norte de Africa. Biblioth. Lichenol., 32: 1-282.

Aceptado para su publicación en Octubre de 1995

Dirección de los autores. Departamento de Biología Vegetal (Botánica), Facultad de Biología, Universidad de Murcia, Campus de Espinardo, 30071 Murcia. 